\title{
Informed Consent in Chronic Schizophrenia?
}

\author{
GARETH H. JONES
}

The current definition of informed consent as used in British psychiatric practice, both for formal and informal patients, is given in the Department of Health's 1993 Code of Practice of the Mental Health Act 1983, and is worth quoting in full.

\section{Consent; the basic principles}

15.12 'Consent' is the voluntary and continuing permission of the patient to receive a particular treatment, based on an adequate knowledge of the purpose, nature, likely effects and risks of that treatment including the likelihood of its success and any alternatives to it. Permission given under any unfair undue pressure is not 'consent'.

This review is aimed at looking at the cognitive processes necessary to give informed consent, and at how intact these processes are in chronic schizophrenia of duration of more than 2 years, and with a deficit of social functioning (as used in various definitions, such as DSM-III-R; American Psychiatric Association, 1987).

The above definition of informed consent is quite a daunting one to the neuropsychologist, involving as it does sufficient working memory to allow the pros and cons of treatment to be held in memory, as also must be the likely natural history of the untreated disease process. Secondly, there must be some element of probabilistic judgement, as the nature of side-effects and indeed of outcome require at least an elementary understanding of probability.

Irwin et al (1985) have written about the difficulties of acutely psychotic patients in understanding informed consent, and examined the abilities of both voluntary and involuntary patients to understand information about anti-psychotic medication. Although most patients stated that they had understood informed consent material, objective ratings did not support this. Impaired understanding was significantly associated with thought disturbance, but surprisingly did not affect acceptance, nor refusal of medication. Legal status was not a significant factor either. However, this was very much a study only of acutely admitted patients, and there is little written on the equivalent in chronic patients.

Rational assessment for the need for treatment

In a recent article, Hatcher (1995) looked at the process of decision analysis in psychiatry. His discussion emphasises that treatment choice is a decision the patient makes on the advice of a doctor, and emphasises the similarity between a medical problem, and gambling on probabilities. Decision analysis is seen as explicit, quantitative, and prescriptive. Numerical values can be put on key uncertainties, and on the personal value of possible outcomes.

A worked example is given concerning prophylactic maintenance anti-psychotics following remission from a first episode of schizophrenia. The risk of recurrence can be estimated from the literature, but the patient's decision will be influenced by how much they value the lower likelihood of recurrence versus the risk of tardive dyskinesia. Perhaps surprisingly, the best decision is very much influenced in the theoretical model by the risk of recurrence without medication, and less so by the value placed on the risk of recurrence without tardive dyskinesia. Hatcher concedes that decision analysis is more likely to be of value in teaching, audit, and research, than in clinical decision taking.

The reader who has got this far, will appreciate that the intellectual processing necessary for the careful analysis of treatment and outcome variables in making the decision to have treatment for a disabling condition such as chronic schizophrenia, which also carries a high death-rate of some $10 \%$ or $15 \%$ as a lifetime risk, is an extremely difficult process.

\section{The 'Sidaway' case}

Scarman (1985), and his fellow Law Lords in the Upper House agreed basically with this sort of analysis, giving legal credence to the processes involved. Mrs Sidaway suffered paralysis following a neurosurgical operation to reduce pressure on nerve-roots in her neck. She argued that she would not have given consent if she had been informed of this very remote, though obviously very serious, complication. The ruling was such that a serious outcome was so remote, that it need not have been raised in the original process of gaining informed consent.

Once again, the patient giving informed consent clearly needs some estimate of probabilities in assessing whether or not the side-effects of treatment 
are serious, and whether or not they are better than being left with the untreated condition. This case also illustrates the importance of changing one's mind as more information is given.

\section{Cognitive abnormalities in chronic schizophrenia}

Crow \& Johnstone (1982) found many cognitive abnormalities in such patients. Their major finding was age disorientation. Some $25 \%$ of their sample were five years or more out in their estimation of their own chronological age. The authors concluded that 'Time stands still', particularly as many such patients reported their age on admission, that is to say about the age of onset, and this sort of finding is particularly reminiscent of patients with severe amnesic syndromes following various sorts of organic brain injury.

As well as age disorientation, there was a severe and generalised impairment of memory and intelligence, including orientation, general knowledge, association learning, remote learning, vocabulary, use of language, visuo-spatial matrix tests, digit symbol substitution tests, and mental tests scores. Their conclusion was that the psychopathological difference between functional and organic psychoses is less maintained in the case of schizophrenia than is sometimes thought to be the case.

Common sense dictates that a patient who does not know their own age would have major difficulties with complex judgements, such as the giving of informed consent.

\section{The Wisconsin Card Sorting Test}

While no specific test is characteristic of chronic schizophrenia (otherwise we would have a diagnostic test), considerable interest of recent years has been taken in this particular test, known to be affected specifically by lesions in the dorso-lateral aspect of the prefrontal cortex. This test is exceedingly tedious to perform and rate, but shortened and simplified versions have become available on micro-computer. Basically, cards with symbols differing in colour, shape, and number are presented sequentially to the patient. The problem that this test identifies, is in noting the switch between concepts outlined above. High perseverative error scores are suggestive of conceptual inflexibility, of particular relevance to the 'Sidaway Case', where the plaintiff claimed that she would have altered her mind if only she had been given extra information.

Not all patients do have problems with the Wisconsin Card Sorting Test, and age, duration of illness and the extent of hospitalisation are factors known to influence results.

It could be argued that these changes occurred secondarily to long-term institutional care. Against this is a recent study of patients with much shorter illnesses. Goldberg et al (1993), in a study of monozygotic twins discordant for schizophrenia, found marked cognitive impairments in the schizophrenic twin. They found that the severity of the declarative learning and memory deficits was strongly associated with the global level of functioning. These were patients with a mean length of illness of only 10.3 years.

Their inability to acquire new information rapidly, access old information propitiously, and divert attention between important environmental stimuli and integrated information processing might make for difficulty 'in even the most routine mental operation of everyday life'. The authors wondered if anticholinergic medication, so commonly prescribed in schizophrenia, might make memory worse, but their basic finding added further weight to the emergent recognition that cognitive impairment is the central aspect of the disease process in schizophrenia and not just an epi-phenomenon of psychopathology, that is to say attention being diverted by hallucinations, thought-blocking and so on.

\section{Are these cognitive deficits amenable to treatment?}

If it could be demonstrated that these cognitive deficits did improve, then a course of treatment might be necessary initially under some compulsory sort of basis, such as is provided by the Mental Health Act, though informed consent might then become easier after clinical recovery. However, Hemsley (1992), reported a permanent weakening of the influence of stored memories of regularities of previous input on current perception, which seemed basic to the chronic schizophrenia. This has been tested by experiments such as latent inhibition, and a key disturbance might well be the failure to relate specific associations to the context in which they occur. Thus the order of presentation of pros and cons might even influence a patient's decision on treatment.

Recent work by Hagger et al (1993) found a modest improvement in cognitive function after clozapine, corresponding roughly to any improvement in psychopathology. However, substantial cognitive impairments remained even after six months of clozapine treatment.

Thus, even with the best technology available today, it is apparent that the cognitive symptoms must as yet be regarded as responding only partially to biological treatment. 
Delahunty et al (1993) attempted specific cognitive flexibility rehabilitation in schizophrenia. This involved using the Wisconsin Card Sorting Test. With a small sample of three, they found some significant gains in the WCST, that were maintained at six month's assessment. However, the improvements were really small, though significant statistically, and there was found to be little improvement on other elements of life-skills or indeed on other psychopathology.

\section{Probabilistic judgements in patients with chronic schizophrenia}

Huq et al (1988) compared deluded subjects with a non-deluded psychiatric control group consisting mostly of anxious patients, as well as a normal control group, on a probabilistic inference task. This was really a ridiculously simple task, involving guessing the proportion of different coloured beads in jam-jars. Beads were mixed in the ratio of $85: 15$, and the subject was blind as to which colour was in the majority. The experimenter picked out beads one at a time and presented them to the subject, who was asked to report when they had reached complete certainty as to which colour was in the majority.

Deluded subjects were found to request less information before reaching a decision, and to express higher certainty levels than either control group. They also exhibited over-confidence on estimates of the probability of a future event. These snap judgements and an over-optimistic view of the future without treatment, are those which are expressed frequently in front of mental health review tribunals.

It should be remembered that normal subjects may well show poor probabilistic judgement. One classic example is the Minimax principle, with people betting on a national lottery because the first prize is so big. The informed statistician would make his decision on the statistical odds, not on the size of the prize. However, normal subjects have no difficulty with Huq's test, which is not one of fine discrimination but of mixtures of 85:15 v. 15:85. This judgement could be seen as a model of the discrimination between major and minor side-effects of a course of treatment.

This study was replicated and extended by Garety et al (1991). They found that after certainty was reached, deluded subjects were much more likely to make a dramatic change in probability estimate following an item of discordant confirmatory information. They felt that this was consistent with those models of schizophrenia which emphasise the greater influence of immediate environmental stimuli compared with the effects of prior learning.
This is of particular interest to the judgement of the 'Sidaway Case', as Mrs Sidaway clearly emphasised that she would have changed her mind with regard to consent to operation if only she had been given extra information.

\section{The intellectual and educational problem}

In their rush to achieve inter-rater reliability, many psychiatrists are now trained in diagnostic systems based ultimately upon Schneider's first rank symptoms. These spectacular delusions and hallucinations do indeed go into remission for many patients with chronic schizophrenia, but the underlying defects of logical thinking which may affect judgement and hence the consent to treatment, do not necessarily respond so well to treatment, nor indeed to the passage of time. Although now unfashionable, Bleuler's four 'A's include asyndetic (illogical) thinking, which is perhaps the most characteristic part of the autism of chronic schizophrenia.

Indeed, there are many comparisons between chronic schizophrenia and frontal lobe impairment that make one think of chronic schizophrenia as a long-term handicapping condition in which the cognitive impairment is truly central.

\section{Conclusion}

Some significant proportion of patients with chronic schizophrenia suffer from defects of cognitive function so grave as to throw into doubt their ability to give informed consent to treatment, in particular the prescription of neuroleptics to reduce the risk of relapse.

The literature is unclear on what proportion are thus affected. However, there are now available a variety of tests, particularly neuropsychological ones, that are rather simplistic models of the processes involved in giving informed consent. The application of such tests may well help the clinician in deciding whether or not such patients are fit to give informed consent, and it is hoped that better experimental models will become available in the future.

\section{Implications for mental health law}

The House of Commons Health Committee rejected in 1993 the concept of a Community Supervision Order on seven grounds, one of which was that patients suffering from mental disorder were normal between episodes and hence should be allowed to exert their normal judgement as to whether or not they choose to continue with preventative treatments. 
From much of the argument above, it is apparent that some patients with chronic schizophrenia have major cognitive impairment, such as to interfere with their judgement, and that this impairment seems relatively enduring.

\section{Acknowledgement}

Thanks are due to Dr David Griffiths of the Division of Psychiatry Ethics Committee for advice and guidance.

\section{References}

Crow, T. J. \& Johnstone, E. C. (1980) Dementia praecox and schizophrenia: was Bleuler wrong? Journal of the Royal College of Physicians, 14, 238-240.

Delahunty, A., Morice, R. \& Frost, B. (1993) Specific cognitive flexibility rehabilitation in schizophrenia. Psychological Medicine, 23, 221-227.
Garety, P. A., Hemsley, D. R. \& Wessley, S. (1991) Reasoning in deluded schizophrenics and paranoid patients. Journal of Nervous and Mental Diseases, 179, 194-201.

Goldrerg, T. E., Torrey, E. F., Gold, T. M. et al (1993) Learning and memory in monozygotic twins discordant for schizophrenia. Psychological Medicine, 23, 71-85.

Hagger, C., Buckley, P., KeNNY, J. T., et al (1993) Improvement in cognitive functions and psychiatric symptoms in treatmentrefractory schizophrenia patients receiving clozapine. British Journal of Psychiatry, 34, 702-712.

HATCHER, S. (1995) Decision analysis in psychiatry. British Journal of Psychiatry, 166, 184-190.

HemsleY, D. R. (1992) Cognitive abnormalities and schizophrenic symptoms (Editorial). Psychological Medicine, 22, 839-842.

HuQ, S. F., Garety, P. A. \& Helmsley, D. R. (1988) Probabilistic judgements in deluded and non-deluded subjects. Quarterly Journal of Experimental Psychology, 40A, 801-812.

Irwin, J., Lovitz, A. A., Marder, S. R., et al (1985) Psychotic patients' understanding of informed consent. American Journal of Psychiatry, 142, 1351-1354.

SCARMAN, LORD (1985) Sidaway v. Gov. of Bethlem Royal Hospital (H. L. (E.)). The Weekly Law Reports, 8 March 1985.

Gareth H. Jones, FRCPsych, Llewelyn Suite, Whitchurch Hospital, Cardiff, CF4 7XB

(First received 22 November 1994, final revision 12 April 1995, accepted 19 May 1995) 